\title{
Cardiac Hydatid Cyst in a Child
}

\author{
A.J. Macedo, M.P. Magalhães, N. Jalles Tavares, L. Bento, F. Sampayo, M. Lima \\ Department of Pediatric Cardiology, Hospital de Santa Marta, Santa Marta Street, 1150 Lisbon, Portugal
}

\begin{abstract}
Cardiac hydatid cyst is a rare disease, especially in children. An 11-year-old boy with a previous anaphylactic reaction and episodes of abdominal pain was admitted for workup of an acquired long systolic murmur. Echocardiographic investigation disclosed a tumor of the right ventricular anterior wall, with multiple loculations. Magnetic resonance imaging characterized it as a multilobular tumor with cyst formation and disclosed another cyst in the right pulmonary artery. With a positive ELISA reaction the child was admitted for surgery with the diagnosis of cardiac and pulmonary hydatid cysts. Cardiac surgery was performed with good results, followed by medical treatment with albendazole.
\end{abstract}

Key words: Cardiac hydatid cyst - Echocardiography — Magnetic resonance imaging — Pediatric cardiology

Hydatid cyst in the heart is uncommon, cardiac involvement is reported in fewer than $2 \%$ of all cases [5, 9]. Diagnosis in children is rare due to the cyst's slow growth [1]. Cardiac cysts are reported to be primary lesions in $80 \%$ of cases $[4,5]$. Most frequently hydatid cysts in children occur within the lung parenchyma. In Portugal the incidence of hydatid cysts is estimated to be approximately 1 per 710,000 [3], and a recent 10 year study reported 20 cases of hydatid cyst in children, but none with cardiac involvement [2]. We report a myocardial hydatid cyst of the right ventricle that was treated successfully with surgery.

\section{Case Report}

An 11-year-old boy was originally seen by our department because of a cardiac murmur. There was no relevant family history, and pregnancy and delivery had been normal with a birth weight of $3400 \mathrm{~g}$. He had been under a regular pediatric surveillance and received the recom-

Correspondence to: A.J. Macedo mended immunizations, BCG included. During infancy he had had several episodes of bronchiolitis and urticaria. Beginning at 2 years of age there was marked alleviation of the respiratory problems, although the atopic dermatitis did not resolve. At the age of 8 years he had been admitted to the Santa Maria University Hospital in Lisbon on an emergency basis owing to a serious anaphylactic crisis of unknown origin. Therapy with intravenous corticosteroids and dopamine was necessary. At the age of 10 he was referred to the same hospital because of acute right upper abdominal pain. No diagnosis was established, and at that time a soft cardiac murmur was found and was interpreted as an innocent murmur. Various episodes of upper abdominal pain, interpreted as psychosomatic problems, took him to various emergency rooms. One month before returning to our department, because of left lower rib pain and dry cough for 2 weeks with no fever his pediatrician diagnosed left-side pneumonia on the basis of a chest radiograph. At that time, a loud cardiac systolic murmur was heard.

At the current admission the clinical examination, including skin, chest, and abdomen, revealed no apparent disease, except for a thrill on the left anterior chest wall. Cardiac auscultation disclosed a loud left sternal ejection murmur with no click and maximal intensity on the third intercostal space. The first heart sound was normal, and there was a normal split second sound with a soft pulmonary component. Blood tests disclosed $16 \%$ eosinophilia. The chest radiograph demonstrated a normal cardiac image and nonspecific bilateral lung opacities. The electrocardiogram (ECG) was normal. On two-dimensional echocardiography a large multicystic mass was apparent within the anterior wall and outflow tract of the right ventricle (Fig. 1). The mass was nonmovable with cardiac cycles, and a Doppler gradient of $40 \mathrm{mmHg}$ was measurable in the outflow tract of the right ventricle. A right ventricular hydatid cyst was suspected. ECG gated magnetic resonance imaging (MRI) demonstrated a lobulated, multiseptated mass in the right ventricle that involved the anterior wall; it exhibited high signal intensity on T2-weighted images (Fig. 2). In addition, a right main pulmonary cyst was shown. The enzyme-linked immunosorbent assay (ELISA) for Echinococcus was positive. Abdominal ultrasonography and brain computed tomography (CT) were normal.

The patient was submitted to open heart surgery, and a large cyst was demonstrated invading the right ventricle anterior wall and extending to the epicardium and the outflow tract (Fig. 3). There was a small rupture on the cyst layer, with an opening to the right ventricular infundibulum. The cyst was resected, and the anterior right ventricle wall closed with direct stretches with Teflon pledget support. The right pulmonary artery cyst was extracted by needle aspiration.

Albendazole $300 \mathrm{mg} /$ day was administered for 4 months after surgery. At 6 and 12 months after surgery the ELISA was repeated, as were clinical and echocardiographic evaluations. The cardiac murmur had almost disappeared, as had the previous right ventricular echocardiographic images, but the ELISA for Echinococcus continued to show 


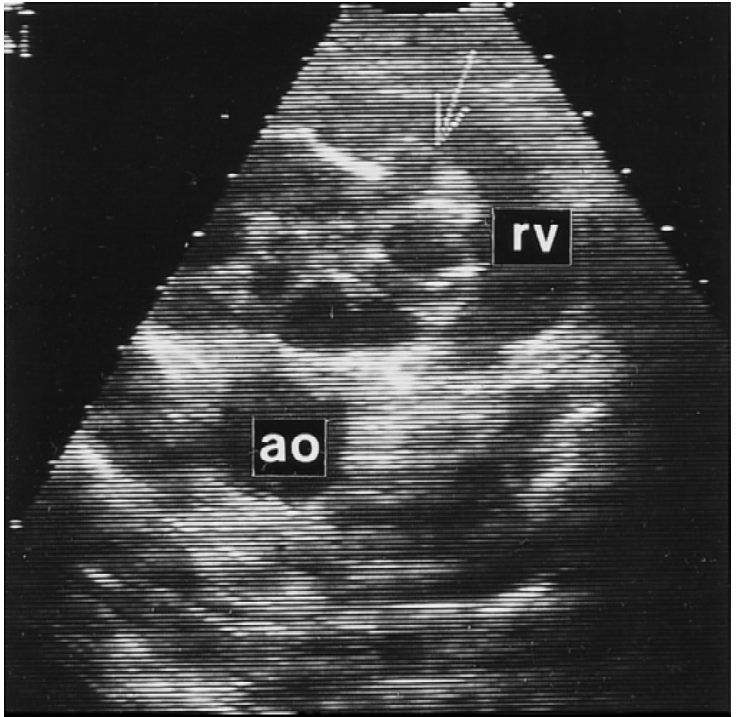

Fig. 1. Transthoracic two-dimensional echocardiography. Parasternal short axis view at the level of the great arteries. A large multicystic mass is observed inside the right ventricle, narrowing the outflow tract. A small opening in the cyst is seen (arrow), confirmed later on surgery (see Fig. 3C). ao, aorta; rv, right ventricle.

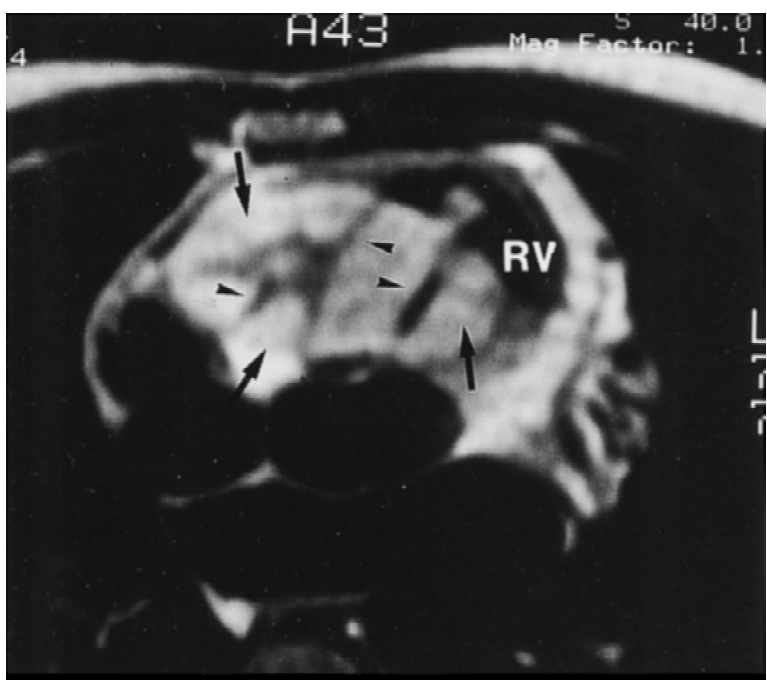

Fig. 2. Magnetic resonance imaging. A lobulated (arrows) and multiseptated (small arrows) mass is demonstrated in the anterosuperior wall of the right ventricle $(R V)$.

high values at 18 months after surgery. MRI was repeated, and a right pulmonary artery cyst was demonstrated, which was resected through a right thoracotomy.

\section{Discussion}

The presence of portacaval and Retzius space shunts in children favors the occurrence of hydatid cysts predomi- nantly in the lung $[2,9]$. In its early stages the Echinococcus larva arrives at the left cardiac cavities through either the pulmonary capillary network or the right atrium due to the patency of the foramen ovale. Reaching the coronary arteries the larva invades the myocardium, requiring 1-5 years to attain adult form. The clinical symptoms induced by hydatid cysts are due to related complications, such as rupture, obstruction, or compression. Although more frequently located on the left ventricle, right ventricular hydatid cysts more frequently rupture, leading to pulmonary embolism, an anaphylactic reaction, or sudden dead. Rupture into the pericardial cavity can also lead to pericarditis, effusion, or cardiac tamponade $[4,5,9]$.

In the case reported here, detection of the cardiac murmur and echocardiography provided the most helpful data. The previous anaphylactic reaction could be interpreted afterward as related to rupture of the cyst into the right ventricle. The ECG was normal, as is usually the case when the cyst is right-sided [9]; the chest radiograph failed to reveal the typical opacity of a pulmonary hydatid cyst. Echocardiographic features of an intracardiac multicystic mass that is relatively fixed associated with eosinophilia and the fact that the child was in an endemic region led to the strong suspicion of a cardiac hydatid cyst. The two-dimensional echocardiographic findings distinguished this mass from other, more frequent cardiac tumors during childhood, namely rhabdomyoma, fibroma, and myxoma [7].

Both CT and MRI have been used to diagnose cardiac hydatid cysts with variable results $[6,12]$. In this case, MRI clearly demonstrated the anatomic location of the mass, its relation to the cardiac chambers, and the presence of a cyst in the pulmonary artery. Tissue characterization on the basis of a T2-weighted scan added some specificity to the diagnosis. After the diagnosis, because of its location and the risk of rupture with consequent pulmonary embolization, surgery was performed on an emergency basis.

About $10 \%$ of all hydatid cysts tend to recur after surgery, but this rate may decrease with proper medical treatment [10]. The ELISA for Echinococcus proved to be a valuable monitoring device to screen for recurrence or undetected cysts. Medical treatment alone is not effective, and surgery is the treatment of choice for cardiac hydatid cyst [8]. Albendazole alone or in combination with praziquantel is effective prophylactic chemotherapy after surgery $[10,11]$.

We conclude that any child with an acquired loud cardiac murmur should be carefully studied. Twodimensional echocardiography, MRI, and ELISA for Echinococcus proved to be excellent methods for establishing the diagnosis in this case, which led to the immediate correct treatment of this rare condition. 


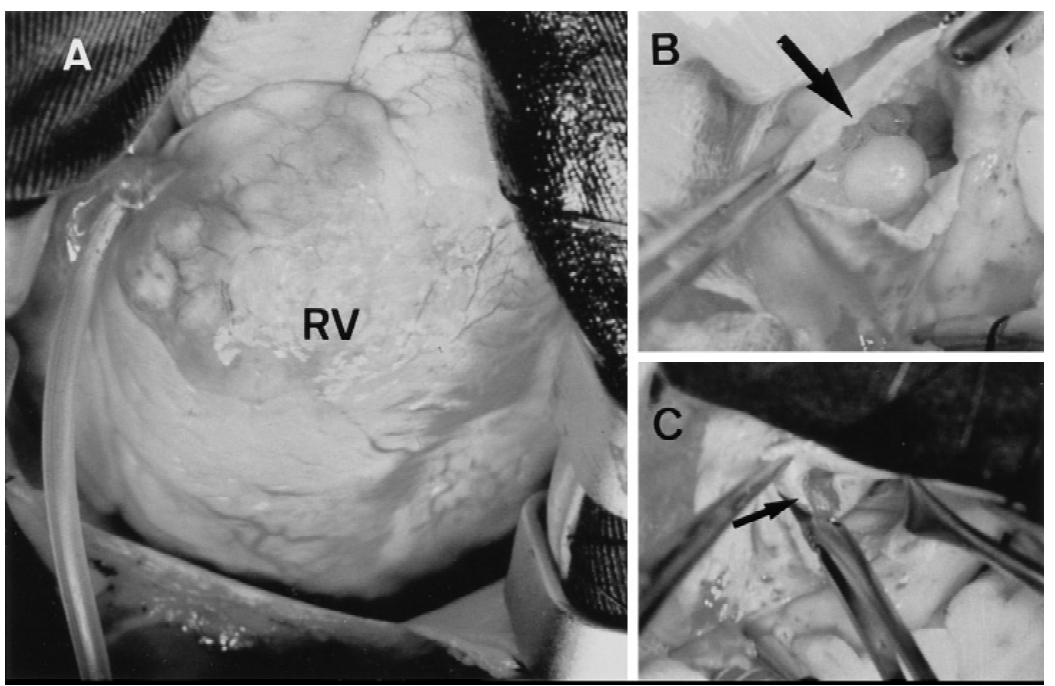

Fig. 3. Intraoperative findings. (A) Anterior wall of the right ventricle, with an irregular aspect caused by the cyst invading the epicardium. $R V$, right ventricle. (B) After opening the wall of the right ventricle, multiple cysts (arrow) can be seen. (C) Ruptured cyst can be seen throughout the pulmonary valve.

\section{References}

1. Ben-Musa AA, Singh H, Shembesh AH, Chugh JC (1990) Cardiac hydatid cyst in a child. Clin Pediatr (Phila) 29:409-411

2. Carvalhal A, Lourenço L (1989) Hidatidose na criança. Arq Hosp Estefania 4:49-58

3. David de Morais JA, Rombert PC, Trinca AT (1985) Subsídios para o conhecimento da epidemiologia da hidatidose rural no alto alentejo. Rev Port Doenc Infecc 1-4:83-94

4. Di Bello R, Menendez H (1963) Intracardiac rupture of hydatid cysts of the heart: a study based on three personal observations and 101 cases in the world literature. Circulation 27:366-374

5. Dighiero J, Canabal EJ, Aguirre CV, Hazan Jacobo, Horjales JO (1958) Echinococcus disease of the heart. Circulation 17:127-132

6. Elkouby A, Vaillant A, Comet B, Malmejac C, Houel J (1990) Cardiac hydatidosis: review of recent literature and report of 15 cases. Ann Chir 44:603-610
7. Farooki ZQ, Adelman S, Green EW (1977) Echocardiographic differentiation of a cystic and a solid tumour of the heart. Am J Cardiol 39:107-111

8. Heilbrunn A, Kittle CF, Dunn M (1963) Surgical management of echinococcal cysts of the heart and pericardium. Circulation 27:219-228

9. Perez-Gomes F, Duran H, Tamames S, Perrote JL, Blanes A (1973) Cardiac echinococcosis: clinical picture and complications. Br Heart J 35:1326-1331

10. Taylor DH, Morris DL (1989) Combination chemotherapy is more effective in postspillage prophylaxis for hydatid disease than either albendazole or praziquantel alone. Br J Surg 76:954

11. Taylor DH, Morris DL, Reffin D, Richards KS (1989) Comparison of albendazole, mebendazole and praziquantel chemotherapy of Echinococcus multilocularis in a gerbil model. Gut 30:1401-1405

12. Vanjak D, Moutaoufik M, Leroy O, Beuscart C, Billiau V (1990) Cardiac hydatidosis: contribution of magnetic resonance imaging: report of a case. Arch Mal Coeur 83:1739-1742 